\title{
Video Article \\ Assessment of the Effects of Endocrine Disrupting Compounds on the Development of Vertebrate Neural Network Function Using Multi-electrode Arrays
}

\author{
Karla R Sanchez ${ }^{1}$, Mahlet D Mersha ${ }^{1}$, Harbinder S Dhillon ${ }^{1}$, Murali K Temburni ${ }^{1}$ \\ ${ }^{1}$ Department of Biological Sciences, Delaware State University
}

Correspondence to: Murali K Temburni at mtemburni@desu.edu

URL: https://www.jove.com/video/56300

DOI: doi: $10.3791 / 56300$

Keywords: Developmental Biology, Issue 134, Bis-phenol-A, C. elegans, chick embryo, EDC, habituation, BPA, embryonic development, embryonic neurons, network activity, synchrony index

Date Published: 4/26/2018

Citation: Sanchez, K.R., Mersha, M.D., Dhillon, H.S., Temburni, M.K. Assessment of the Effects of Endocrine Disrupting Compounds on the Development of Vertebrate Neural Network Function Using Multi-electrode Arrays. J. Vis. Exp. (134), e56300, doi:10.3791/56300 (2018).

\section{Abstract}

Bis-phenols, such as bis-phenol A (BPA) and bis-phenol-S (BPS), are polymerizing agents widely used in the production of plastics and numerous everyday products. They are classified as endocrine disrupting compounds (EDC) with estradiol-like properties. Long-term exposure to EDCs, even at low doses, has been linked with various health defects including cancer, behavioral disorders, and infertility, with greater vulnerability during early developmental periods. To study the effects of BPA on the development of neuronal function, we used an in vitro neuronal network derived from the early chick embryonic brain as a model. We found that exposure to BPA affected the development of network activity, specifically spiking activity and synchronization. A change in network activity is the crucial link between the molecular target of a drug or compound and its effect on behavioral outcome. Multi-electrode arrays are increasingly becoming useful tools to study the effects of drugs on network activity in vitro. There are several systems available in the market and, although there are variations in the number of electrodes, the type and quality of the electrode array and the analysis software, the basic underlying principles, and the data obtained is the same across the different systems. Although currently limited to analysis of two-dimensional in vitro cultures, these MEA systems are being improved to enable in vivo network activity in brain slices. Here, we provide a detailed protocol for embryonic exposure and recording neuronal network activity and synchrony, along with representative results.

\section{Video Link}

The video component of this article can be found at https://www.jove.com/video/56300/

\section{Introduction}

4,4'-Isopropylidenediphenol, commonly referred to as Bisphenol-A or BPA, is an anti-oxidant additive found in a wide variety of polycarbonate and epoxy resin based products ranging from thermal paper, CDs, and shatter proof glass, to the inside coating of beverage cans. Although BPA has been known to be an endocrine disrupting agent that mimics estrogen ${ }^{1}$, studies on its ill effects due to everyday casual exposure to BPA have mostly come out in the last 10-15 years. The consequences of even low levels of BPA exposure are most profound in early developmental periods, including during embryogenesis through exposure of mothers ${ }^{2,3}$. In utero exposure of female embryos to endocrine disrupting chemicals has also been linked to increased disease susceptibility from vaginal to breast cancer ${ }^{4,5}$. Animal studies have demonstrated exposure to BPA leads to atypical brain structure and functional abnormalities manifested in behavior ${ }^{6}$. As a consequence, the use of BPA in infant feeding bottles has been banned by most regulatory agencies in Europe and North America, including the FDA. To comply with regulations, many manufacturers have switched to 4,4'-sulfonyldiphenol or bisphenol-S (BPS). Based on the fact that BPA and BPS are structural analogues and that recent reports showed comparable potency of BPS in estrogenic transcription ${ }^{7}$, it is important to study the toxicity of this compound relative to BPA

Here we describe a protocol to test the effects of BPA (and other EDCs) on networks of neurons using a vertebrate model, the chick embryo. In vitro cultures of neurons form synaptic contacts and generate action potentials (also called spikes). The spiking activity of these cultures can be recorded using multi-electrode array (MEA) systems. Spikes are considered to be in synchrony when they occur within $5 \mathrm{~ms}$ of each other. Initial random spiking activity that eventually synchronizes is a key feature of developing neuronal networks ${ }^{8,9}$. Synchrony can be measured using various methods and several algorithms are described in the literature ${ }^{9,10,11}$. In our analyses, we use an algorithm developed by Paiva and co-workers ${ }^{12}$ which is integrated into the recording software which drives the MEA acquisition system. The robust spiking activity of embryonic chick neurons provides a prototype for studying the effect of BPA on neural network activity ${ }^{13}$. Using multi-electrode arrays to record spiking activity, we observed that BPA exposure inhibits the development of neuronal spiking synchrony ${ }^{9,14}$. Here, we provide a detailed methodology for studying BPA exposure on chick embryo neurons in culture along with representative results on the development of neuronal spiking synchrony in chick embryonic cultures. 


\section{Protocol}

The protocol given below has been standardized to test the effects of BPA exposure during (early) embryogenesis, and may be modified for use with BPS, BPF, or other EDC in chick embryonic neurons.

This protocol follows the institutional policies of Delaware State University and the NIH policy for avian embryos. The protocol also is in confirmation with DSU's material and chemical safety guidelines.

\section{Material Setup}

1. Dissolve BPA (m.w. 228.29) in $10 \%$ ethanol $(\mathrm{v} / \mathrm{v})$ in water to make a $1 \mathrm{mM}$ stock solution $(0.23 \mathrm{mg}$ per $\mathrm{mL}$ of $10 \%$ ethanol). Make subsequent dilutions $(0.1 \mu \mathrm{M}, 0.5 \mu \mathrm{M}, 1 \mu \mathrm{M}, 5 \mu \mathrm{M}$, and $10 \mu \mathrm{M})$ in neurobasal medium.

Caution: BPA is an environmental toxin and care should be exercised when handling the powder.

2. Incubate chick eggs in a commercially available tabletop egg incubator at $37^{\circ} \mathrm{C}$. Incubate eggs for 7 days for E7 embryos.

3. Prepare MEAs. Sterilize the required number of MEAs by soaking in $70 \%$ ethanol for 3 to $4 \mathrm{~h}$ and then rinse three times in approximately 10 $\mathrm{mL}$ of sterile distilled water in the BSLII hood.

NOTE: The MEAs contain 64 nanoporous platinum electrodes arranged in an $8 \times 8$ grid. The electrode diameter is $30 \mu \mathrm{m}$ and the spacing between the electrodes is $200 \mu \mathrm{m}$. Do not use denatured ethanol for sterilizing the MEAs as this will lead to corrosion of the MEA.

4. Place the MEAs inside a sterile container with lid (to maintain sterile conditions) and move to a table top incubator. Bake for at least 6 hours at $55^{\circ} \mathrm{C}$. This step is critical for proper sterilization and the temperature should not exceed $60{ }^{\circ} \mathrm{C}$. Maintaining sterility, move the dish containing MEAs to the BSLII hood for storage until further use.

NOTE: We use an oven safe glass baking dish with plastic lid and surface sterilize it with $70 \%$ ethanol and place in the BSLII hood for drying.

5. Aliquot the extra cellular matrix ECM solution. Thaw the vial at $4{ }^{\circ} \mathrm{C}$ overnight and freeze $100 \mu \mathrm{L}$ aliquots at $-20^{\circ} \mathrm{C}$.

NOTE: ECM is shipped frozen. ECM should not be brought to room temperature until ready to coat as it polymerizes at room temperature and, once polymerized, it is impossible to coat. ECM without growth factors is preferred to prevent additional effects due to unknown factors

6. Sterilize all dissection instruments (Dumont \#5 forceps, curved forceps, small scissors, and spring scissors) and self-sealing material coated dissection dishes with $70 \%$ ethanol and let them dry in the dissection hood.

\section{Chick Embryonic Neuron Culture and Network Activity}

1. On the day of plating, remove a vial of ECM from the $-20^{\circ} \mathrm{C}$ freezer, spray with $70 \%$ ethanol, and place on ice. Dilute to $25 \%$ by adding 300 $\mu \mathrm{L}$ cold neurobasal medium inside BSLII hood.

2. Using a P200 pipetteman add $100 \mu \mathrm{L}$ of $25 \%$ ECM to the center of the MEA taking care not to touch the electrodes and remove immediately leaving a thin film on the surface. Cover the MEA and place in the $\mathrm{CO}_{2}$ incubator $\left(37^{\circ} \mathrm{C}\right.$ and $\left.5 \% \mathrm{CO}_{2}\right)$ until ready to plate the neurons.

3. Sterilize the outer shell of an E7 egg (embryonic day 7, incubated at $37^{\circ} \mathrm{C}$ for 7 days) with $70 \%$ ethanol. Decapitate the embryo into a Sylgard bottom dissection dish containing cold sterile Hank's Balanced Salts Solution (HBSS) without calcium. Cut around the eyes and remove the eyeballs. Using DuMont \#5 fine forceps and spring scissors make an incision on the ventral side and remove the outer layers of skin to expose the forebrain and optic tectum. Peel and remove the pial membrane carefully. Transfer the forebrain into another petri dish and cut it into small pieces of about $2 \mathrm{~mm}$ with spring scissors.

NOTE: There should not be any blood vessels attached to the forebrain after removal of the pial membrane. If available, it is preferable to perform the dissection in a sterile dissection hood, although we have obtained fairly good results when dissection is performed outside a hood as long as the instruments and dissection area are thoroughly sterilized with $70 \%$ ethanol.

4. Using a sterile transfer pipette, collect the pieces of forebrain into a $15 \mathrm{~mL}$ centrifuge tube and remove as much of the HBSS as possible after letting the pieces of forebrain sink to the bottom of the centrifuge tube.

5. Add $1 \mathrm{~mL}$ of $0.05 \%$ Trypsin/EDTA (pre-warmed to $37^{\circ} \mathrm{C}$ ) and incubate at $37^{\circ} \mathrm{C}$ for 15 minutes.

6. Using a Pasteur pipette, carefully remove trypsin without disturbing the pieces of tissue and add $1 \mathrm{~mL}$ neurobasal medium. Let the pieces of tissue sink to the bottom and remove the medium. Repeat this wash once more. This step is to wash off the trypsin/EDTA.

7. Add $2 \mathrm{~mL}$ of neurobasal medium and start triturating. Trituration involves taking a sterile fire-polished Pasteur pipette and passing the tissue through it several times gently until no more pieces of tissue are seen. If any pieces remain after repeated passes, just leave them to settle to the bottom. NOTE: Avoid frothing while triturating - if froth does form, stop and remove by aspiration.

8. Dilute the re-suspended cells 1:10 with neurobasal medium and count viable cells using Trypan Blue dye and a hemocytometer. Mix $50 \mu \mathrm{L}$ of the diluted cells with $50 \mu \mathrm{L}$ of Trypan Blue solution in a $0.5 \mathrm{~mL}$ centrifuge tube. Add $10 \mu \mathrm{L}$ to the hemacytometer after placing on the coverslip and count the bright clear cells (blue cells are dead and should not be counted). NOTE: Typical cell numbers from a single E7 optic tectum range between 1 and $5 \times 10^{7}$ cells $/ \mathrm{mL}$.

9. Plate the dissociated cells on ECM coated MEAs at a density of 2,200 cells/square $\mathrm{mm}$. For our MEA system, this translates to approximately 130,000 cells per MEA. Add neurobasal medium to bring the volume in the MEA to $1 \mathrm{~mL}$ and place MEAs in the $\mathrm{CO}_{2}$ incubator overnight for cell attachment and neurite extension (Figure 1).

NOTE: For MEAs of different areas and electrode numbers, different cell densities could be tried - in general, higher cell density results in greater network activity, but also leads to shorter lived cultures.

10. The next day, add BPA to a final concentration of $10 \mu \mathrm{M}$ in neurobasal medium from the $1 \mathrm{mM}$ stock made in step 1.1 to the treated MEA. To the control MEA, add the same volume of the $10 \%$ ethanol in water $(\mathrm{v} / \mathrm{v})$ solution.

11. Replace spent medium with neurobasal medium containing $10 \mu \mathrm{M}$ BPA every other day until 7 days in vitro (7DIV) and every day thereafter, as the network activity increases.

NOTE: As the network activity increases, metabolic activity increases and media changes need to be more frequent. Do not let the medium turn orange. 


\section{Recording Neuronal Network Activity}

1. On the day of acquisition, exchange the culture medium with fresh neurobasal medium and return the $M E A s$ to the $\mathrm{CO}_{2}$ incubator for a minimum of $2 \mathrm{~h}$ before recording. Start the recording software and set the temperature of the MEA to $37^{\circ} \mathrm{C}$ by clicking on the temperature icon (Supplemental Figure 3.1a-b).

NOTE: Recording can begin as early as day 3 of plating and can continue as long as the cultures are alive.

2. Set up the acquisition parameters by right-clicking on the "Muse" icon (under Streams in the left window panel) and select "Add Processing" and "Spike Detector" and click OK in the pop up window. "Spike Detector (6 x STD)" will appear below the file name (Supplemental Figure 3.2a-b).

3. Next right click on the Spike Detector, select "Add Processing" and "Burst Detector" and click OK in the pop up window. "Burst Detector" (ISI) will appear below the Muse icon (Supplemental Figure 3.3a-b).

4. Next right click on Burst Detector, select "Add Processing" and "Neural Statistics Compiler." In the pop up window, ensure that File Header, Aggregated Well Statistics and Synchrony are selected. Click OK. "Statistics Compiler" will appear below (Supplemental Figure 3.4a-b). NOTE: This will set the adaptive threshold crossing (6x STD filter), the inter-spike interval at $100 \mathrm{~ms}$ with a minimum number of spikes at 5 , the mean firing rate detection at $10 \mathrm{~s}$ with a synchrony parameter at $20 \mathrm{~ms}$, and the minimum spike rate at $0.083333 \mathrm{spikes}$ per min.

5. Set up the scheduled recording for the recording time of 5 minutes $(300,000 \mathrm{~ms})$ by clicking on the clock icon at the bottom. This is achieved by changing the information in the setting section to record every 5.1 minutes and record for 5 minutes. This will be started immediately and will be executed once (Supplemental Figure 3.5).

6. Ensure that the temperature of the MEA has reached $37^{\circ} \mathrm{C}$ before moving the MEA. Remove the MEA from the incubator, place it on the recording unit, and lock down the MEA. Start recording the network activity by clicking on the start record in the scheduled recording section or the record icon in the "File Play" window. Typically, a recording time of 5 minutes $(300,000 \mathrm{~ms})$ is sufficient, although longer recordings of up to 10 minutes can be obtained.

7. After recording, return the MEA to the incubator. NOTE: Care must be taken to maintain sterility and to minimize the time the MEA is outside the incubator.

\section{Data Analysis}

1. Analysis of the raw data can be done offline using the recording software. Click on the File pull down menu and open recording. Select the raw data file to be analyzed. Replay the raw recordings to capture the required spiking parameters.

2. To obtain the Average Number of Spikes, use the Spike Detector module and for obtaining Synchrony Index, use the Neural Statistics Compiler module.

3. Load the Spike Detector, Burst Detector and Statistics Compiler Modules as before (Section 3).

4. From the pull-down menus within the Spike Detector, Burst Detector, and Statistics Compiler module windows, select Spike List, Network Burst List, and Advanced Metrics, respectively.

5. Click the record button to start recording the data file which will create a .csv file in the directed folder. The spike parameters - the total number of spikes and the Synchrony Index - will be recorded in the .csv file.

NOTE: Real time analysis while recording raw data is not recommended as this could consume processor time and computational resources.

\section{Representative Results}

We examined the effect of BPA on synchrony development in chick embryo neuronal cultures. Synchronization of spiking activity is an indicator of the normal development of neuronal networks in vitro. When two spikes occur within $5 \mathrm{~ms}$ of each other, they are considered synchronous. In vitro neuronal cultures initially display random spiking activity — spikes occur randomly and the inter-spike intervals show a normal distribution. As the cultures mature, the spiking activity becomes more synchronized and the inter-spike intervals are constant. Synchronous spiking activity of a culture was quantitated by cross-correlation analysis of the spike times using recording software ${ }^{12}$ to derive a Synchrony Index (SI). We found that BPA exposure adversely affects network activity development by reducing spiking activity and the synchrony index (Figure $2 \mathbf{a}$ and 2b). In summary, we have shown that the detrimental effects of BPA exposure on an embryo impacts its development, including neuronal function that can be assessed through synchrony development in chick neurons in culture. 

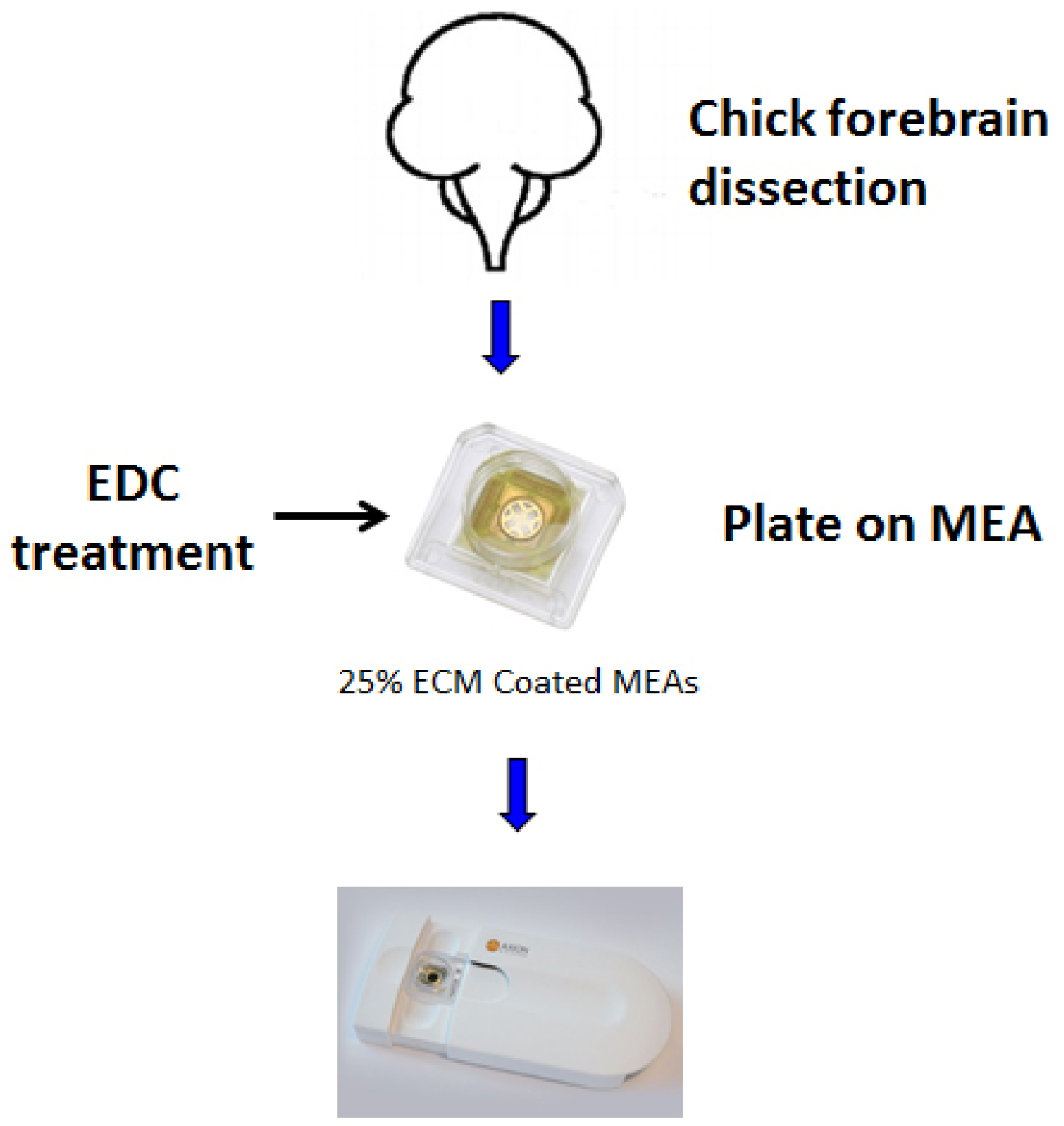

\section{Electrophysiology on MEA recording system after 7 days}

Figure 1: Schematic of the protocol depicting the steps from dissection of chick forebrain to dissociation, plating, and recording of network activity. Please click here to view a larger version of this figure. 

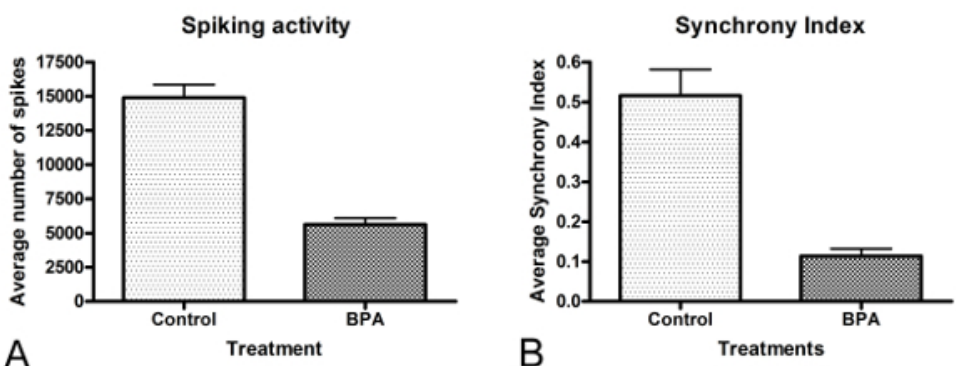

Figure 2: (A) The average number of spikes is significantly lower in BPA treated E7 chick forebrain cultures when compared to control cultures. The average number of spikes was extracted using the recording software. The means (control, 14,890 $\pm 949.0, \mathrm{~N}=15$ and BPA, 5,624 \pm 465.9, $\mathrm{N}=22$ ) were significantly different (unpaired t-test, $\mathrm{p}<0.0001)$. (B) The average synchrony index (SI) is significantly lower in BPA treated E7 chick forebrain cultures when compared to control cultures. The SI was extracted using the Neural Statistics Compiler module within the recording software. The SI means (control, $0.5159 \pm 0.06547 \mathrm{~N}=15$ and BPA, $0.1140 \pm 0.01840 \mathrm{~N}=22$ ) were significantly different (unpaired ttest, $p<0.0001$ ). Error bars depict standard deviation. Please click here to view a larger version of this figure.

\section{Discussion}

Phases of rapid growth and development such as embryogenesis are particularly vulnerable to the detrimental effects of various endocrinedisrupting compounds including BPA. We provide detailed protocols for studying the effects of BPA exposure in an in vitro vertebrate neuronal network model. Using this protocol, we established that BPA lowers the spike rate as well as the synchrony index in the developing neuronal networks (Figure 2a-b).

Our methodology was designed to study BPA exposure during early embryogenesis and can be easily adapted to study the effects of other EDCs. Embryonic neuronal cultures from the chick are relatively easy to establish compared to other vertebrate models, including the rat or mouse. Moreover, there is no requirement for a separate animal room - a simple incubator on a laboratory bench is sufficient to carry out these assays. We describe the use of a multi-electrode system (MEA) for assessing the effect of the EDC, BPA, on network activity. The protocols described here can be applied to other systems. A critical aspect of this protocol is maintenance of sterility. This includes dissection of the embryo under sterile conditions - sterilizing instruments with $70 \%$ ethanol and use of sterile Hank's Balanced Salts Solution is sufficient. As the data is collected over a period of weeks, it is critical to maintain sterile handling of the MEAs. The neuronal cultures once established can be cultured long-term - up to three months - and are thus useful for studying long-term exposure to EDCs and other compounds on neuronal networking. Another important aspect of this protocol is the time from dissection to plating. The optimal time is 30 min, including the incubation of the tissue with trypsin. The longer the time taken, the poorer the survival of the cells.

We describe here a basic protocol that can be applied to the assessment of any chemical or drug that affects behavior and neural function. Here, we have used a cell density of 2,200 cells per $\mathrm{mm}^{2}$; however, this can be modified and other cell densities can be used. In general, we have found that increasing cell density increases network activity - more spikes in a shorter time. The method described, although very useful in assessing effects of chemicals on network activity does have limitations. One of the greatest limitations of the method is that these in vitro cultures are two dimensional may not reflect the three-dimensional architecture of the vertebrate brain. This can be overcome by using slice recordings. Another alternative is to apply the treatments to the developing chick embryo by means of a small window cut on the broad end of the egg ${ }^{15}$, dissect the brain at the end of the treatment regimen, make thick sections on a vibratome, and place onto the MEA for recording network activity.

Our protocol is significant in that it enables examination of the effect of EDCs on the development of network activity and offers the exploration of a mechanistic basis for the effects of these chemicals.

\section{Disclosures}

The authors declare that they have no competing interests.

\section{Acknowledgements}

This study is supported by the NSF (HBCU-UP Research Initiation Award, HRD 1401426 and EPSCoR EPS-0814251) and NIH (COBRE 1P20GM103653-01A1). K.S. is supported by a fellowship from the Delaware INBRE-III 6404.

\section{References}

1. Anahara, R., Yoshida, M., Toyama, Y., Maekawa, M., Masayuki, K., Ishino, F., Toshimori, K., et al. Estrogen agonists, 17 beta-estradiol, bisphenol A, and diethylstilbestrol, decrease cortactin expression in the mouse testis. Arch. of Histol. Cytol. 69(2), 101-107 (2006).

2. Dodds, C. Synthetic oestrogens. Br. Med. Bull., 11(2):131-134 (1955).

3. Grignard, E., Lapenna, S., Bremer, S. Weak estrogenic transcriptional activities of Bisphenol A and Bisphenol S. Toxicology In Vitro., 26(5):727-31 (2012)

4. Herbst, A.L., Ulfelder, H., Poskanzer, D.C. Adenocarcinoma of the vagina: association of maternal stilbestrol therapy with tumor appearance in young women. New Eng. J. Med., 284:878-881 (1971). 
5. Jenkins, S., Raghuraman, N., Eltoum, I., Carpenter, M., Russo, J., Lamartiniere, C.A. Oral exposure to bisphenol A increases dimethylbenzanthracene-induced mammary cancer in rats. Environ. Health Persp. 117(6):910-915 (2009).

6. Okada, A., \& Kai, O. Effects of estradiol-17beta and bisphenol A administered chronically to mice throughout pregnancy and lactation on the male pups' reproductive system. Asian J Androl., 10(2), 271-276 (2008).

7. Palanza, P., Gioiosa, L., Vom Saal, F.S., \& Parmigiani, S. Effects of developmental exposure to bisphenol A on brain and behavior in mice. Environ. Res., 108(2), 150-157 (2008).

8. Mimoto, A., Fujii, M., Usami, M., Shimamura, M., Hirabayashi, N., Kaneko, T., et al. Identification of an estrogenic hormone receptor in Caenorhabditis elegans. Biochem. Biophys. Res. Commun. 364(4), 883-888 (2007).

9. Allard, P., Colaiacovo, M. Bisphenol A impairs the double-strand break repair machinery in the germLine and causes chromosome abnormalities. Proceedings Natl. Acad. Sci. 107(47), 20405-20410 (2010).

10. Mersha, M.D., Patel, B.M., Patel, D., Richardson, B.N., \& Dhillon, H.S. Effects of BPA and BPS exposure limited to early embryogenesis persist to impair non-associative learning in adults. Behav. Brain Funct. : BBF. 11, 27 (2015).

11. Chen, Y., Shu, L., Qiu, Z., Lee, D.Y., Settle, S.J., et al. Exposure to the BPA-Substitute Bisphenol S Causes Unique Alterations of GermLine Function. PLOS Genetics. 12(7), e1006223 (2016).

12. Shein Idelson, M., Ben-Jacob, E., and Hanein, Y. Innate Synchronous Oscillations in Freely-Organized Small Neuronal Circuits. PLoS ONE. 5(12), e14443 (2010).

13. Baltz, T., Herzog, A., and Voigt, T. Slow Oscillating Population Activity in Developing Cortical Networks: Models and Experimental Results. J. Neurophysiol. 106(3), 1500-1514 (2011).

14. Pettmann, B., Louis, J.C., and Sensenbrenner, M. Morphological and Biochemical Maturation of Neurones Cultured in the Absence of Glial Cells. Nature.281(5730), 378-80 (1979).

15. Zhang, H., Wu, C.Y., Wang, W., and Harrington, M.A. Interneuronal Synapses Formed by Motor Neurons Appear to Be Glutamatergic. NeuroReport.,. 22(16), 809-813 (2011).

16. Paiva, A.R.C., Park, I., and Príncipe, J.C.. A Comparison of Binless Spike Train Measures." Neural Comp. and Appl. 19 (3), 405-19 (2010).

17. Cretu, A., Fotos, J.S., Little, B.W., and Galileo, D.S.. Human and Rat Glioma Growth, Invasion, and Vascularization in a Novel Chick Embryo Brain Tumor Model. Clin Exp Metastasis. 22, 225-236 (2005).

18. Chiappalone, M., et al. Dissociated cortical networks show spontaneously correlated activity patterns during in vitro development. Brain Res., 93 (2006).

19. $\mathrm{Li}, \mathrm{X}$., et al. Long-term recording on multi-electrode array reveals degraded inhibitory connection in neuronal network development. Biosens Bioelectron., 22(7), pp.1538-43 (2007). 